\title{
Cyclaniliprole (Pesticides)
}

\author{
Summary
}

\author{
Food Safety Commission of Japan
}

The Food Safety Commission of Japan (FSCJ) conducted a risk assessment of cyclaniliprole (CAS No. 1031756-98-5), an anthranilamide insecticide, based on results from various studies. Major treatment-related effects of cyclaniliprole were observed on liver (increased liver weight and increased alkaline phosphatase) in dogs and on thyroid (hypertrophy of follicular epithelial cells) in rats. None of neurotoxicity, carcinogenicity, reproductive toxicity, teratogenicity, immunotoxicity and genotoxicity were observed. Based on the results from various studies, only parent cyclaniliprole was identified as the relevant substance to the residue definition for dietary risk assessment in agricultural products. The lowest no-observedadverse-effect level (NOAEL) obtained in all the toxicity studies was $1.29 \mathrm{mg} / \mathrm{kg}$ bw/day in a one-year chronic toxicity study in dogs. FSCJ has established an acceptable daily intake (ADI) of $0.012 \mathrm{mg} / \mathrm{kg}$ bw/day, by applying a safety factor of 100 to the NOAEL. FSCJ judged it unnecessary to specify an acute reference dose (ARfD) since no adverse effects, possibly elicited by a single oral administration, was observed.

\section{Conclusion in Brief}

The Food Safety Commission of Japan (FSCJ) conducted a risk assessment of cyclaniliprole (CAS No. 1031756-98-5), an anthranilamide insecticide, based on results from various studies.

The data used in the assessment include the fate in animals (rats, goats and chickens), fate in plants (apples and lettuce), residues in crops, subacute toxicity (rats, mice and dogs), subacute neurotoxicity (rats), chronic toxicity (rats and dogs), carcinogenicity (rats and mice), two-generation reproductive toxicity (rats), developmental toxicity (rats and rabbits), genotoxicity, and immunotoxicity (mice).

Major treatment-related effects of cyclaniliprole were observed on liver (increased liver weight and increased alkaline phosphatase) in dogs and on thyroid (hypertrophy of follicular epithelial cells) in rats. None of neurotoxicity, carcinogenicity, reproductive toxicity, teratogenicity, immunotoxicity and genotoxicity were observed.
Based on the results from various studies, only parent cyclaniliprole was identified as the relevant substance to the residue definition for dietary risk assessment in agricultural products.

The lowest no-observed-adverse-effect level (NOAEL) obtained in all the toxicity studies was $1.29 \mathrm{mg} / \mathrm{kg}$ bw/day in a one-year chronic toxicity study in dogs. FSCJ has established an acceptable daily intake (ADI) of $0.012 \mathrm{mg} / \mathrm{kg}$ bw/ day, by applying a safety factor of 100 to the NOAEL.

FSCJ judged it unnecessary to specify an acute reference dose (ARfD), since no adverse effects, possibly elicited by a single oral administration, was observed.

Published online: 30 March 2017

This is an English translation of excerpts from the original full report (October 2016-FS/640/2016). Only original Japanese texts have legal effects.

The original full report is available in Japanese at http://www.fsc.go.jp/fsciis/attachedFile/download?retrievalId=kya20160511024\&file $\mathrm{Id}=201$

Acknowledgement: FSCJ wishes to thank the members of Expert Committee on Pesticides for the preparation of the original full report. Suggested citation: Food Safety Commission of JAPAN. Cyclaniliprole: Summary. Food Safety. 2017; 5 (1): 29-30. doi:10.14252/ foodsafetyfscj.2016032s 
Levels relevant to toxicological evaluation of cyclaniliprole

\begin{tabular}{|c|c|c|c|c|c|}
\hline Species & Study & $\begin{array}{c}\text { Dose } \\
(\mathrm{mg} / \mathrm{kg} \text { bw/day })\end{array}$ & $\begin{array}{c}\text { NOAEL } \\
(\mathrm{mg} / \mathrm{kg} \text { bw/day) }\end{array}$ & $\begin{array}{c}\text { LOAEL } \\
(\mathrm{mg} / \mathrm{kg} \text { bw/day) }\end{array}$ & Critical endpoints ${ }^{1)}$ \\
\hline \multirow{7}{*}{ Rat } & $\begin{array}{l}90 \text {-day subacute } \\
\text { toxicity study }\end{array}$ & $\begin{array}{l}0,600,6,000,20,000 \mathrm{ppm} \\
\text { M: } 0,39.9,402,1,330 \\
\text { F: } 0,43.3,467,1,590\end{array}$ & $\begin{array}{l}\text { M: } 1,330 \\
\text { F: } 1,590\end{array}$ & $\begin{array}{l}\text { M: - } \\
\text { F: - }\end{array}$ & $\begin{array}{l}\text { F/M: No toxicological } \\
\text { effects }\end{array}$ \\
\hline & $\begin{array}{l}\text { 90-day subacute } \\
\text { neurotoxicity study }\end{array}$ & $\begin{array}{l}0,600,3,100,16,000 \mathrm{ppm} \\
\text { M: } 0,40,204,1,090 \\
\text { F: } 0,49,240,1,280\end{array}$ & $\begin{array}{l}\text { M: } 1,090 \\
\text { F: } 1,280\end{array}$ & $\begin{array}{l}\text { M: - } \\
\text { F: - }\end{array}$ & $\begin{array}{l}\text { F/M: No toxicological } \\
\text { effects } \\
\text { (Not neurotoxic) }\end{array}$ \\
\hline & $\begin{array}{l}\text { One-year chronic } \\
\text { toxicity study }\end{array}$ & $\begin{array}{l}0,200,2,000,6,000, \\
20,000 \mathrm{ppm} \\
\text { M: } 0,9.21,89.6,277,955 \\
\text { F: } 0,11.7,117,358,1,210\end{array}$ & $\begin{array}{l}\text { M: } 955 \\
\text { F: } 1,210\end{array}$ & $\begin{array}{l}\text { M: - } \\
\text { F: - }\end{array}$ & $\begin{array}{l}\text { F/M: No toxicological } \\
\text { effects }\end{array}$ \\
\hline & $\begin{array}{l}\text { Two-year } \\
\text { carcinogenicity } \\
\text { study }\end{array}$ & $\begin{array}{l}0,200,2,000,6,000 \\
20,000 \mathrm{ppm} \\
\text { M: } 0,7.93,82.5,249,834 \\
\text { F: } 0,10.3,103,306,1,040\end{array}$ & $\begin{array}{l}\text { M: } 249 \\
\text { F: } 1,040\end{array}$ & $\begin{array}{l}\text { M: } 834 \\
\text { F: - }\end{array}$ & $\begin{array}{l}\text { M: Hypertrophy of } \\
\text { thyroid follicular } \\
\text { epithelial cell } \\
\text { F: No toxicological } \\
\text { effects } \\
\text { (Not carcinogenic) }\end{array}$ \\
\hline & \multirow{2}{*}{$\begin{array}{l}\text { Two-generation } \\
\text { reproductive toxicity } \\
\text { study }\end{array}$} & \multirow[t]{2}{*}{$\begin{array}{l}\text { 0, 500, 3,000, 20,000 ppm } \\
\text { PM: 0, 34.9, 207, 1,410 } \\
\text { PF: 0, 39.2, 228, 1,590 } \\
\text { F }_{1} \mathrm{M}: 0,41.2,245,1,680 \\
\mathrm{~F}_{1} \mathrm{~F}: 0,45.6,274,1,840\end{array}$} & $\begin{array}{l}\text { Parent: } \\
\text { PM: } 1,410 \\
\text { PF: } 1,590 \\
\mathrm{~F}_{1} \mathrm{M}: 1,680 \\
\mathrm{~F}_{1} \mathrm{~F}: 1,840 \\
\end{array}$ & $\begin{array}{l}\text { Parent: } \\
\text { PM: - } \\
\text { PF: - } \\
\mathrm{F}_{1} \mathrm{M}:- \\
\mathrm{F}_{1} \mathrm{~F}:-\end{array}$ & $\begin{array}{l}\text { Parent } \\
\text { F/M: No toxicological } \\
\text { effects }\end{array}$ \\
\hline & & & $\begin{array}{l}\text { Offspring: } \\
\text { PM: } 1,410 \\
\text { PF: } 1,590 \\
\mathrm{~F}_{1} \mathrm{M}: 1,680 \\
\mathrm{~F}_{1} \mathrm{~F}: 1,840 \\
\end{array}$ & $\begin{array}{l}\text { Offspring: } \\
\text { PM: - } \\
\text { PF: - } \\
\mathrm{F}_{1} \mathrm{M}:- \\
\mathrm{F}_{1} \mathrm{~F}: \text { - }\end{array}$ & $\begin{array}{l}\text { Offspring } \\
\text { F/M: No toxicological } \\
\text { effects } \\
\text { (No effect on reproduc- } \\
\text { tion) }\end{array}$ \\
\hline & $\begin{array}{l}\text { Developmental } \\
\text { toxicity study }\end{array}$ & $0,100,300,1,000$ & $\begin{array}{l}\text { Maternal: } 1,000 \\
\text { Embryo/fetus: } 1,000\end{array}$ & $\begin{array}{l}\text { Maternal: - } \\
\text { Embryo/fetus: - }\end{array}$ & $\begin{array}{l}\text { Maternal: No toxicologi- } \\
\text { cal effects } \\
\text { Embryo/fetus: No } \\
\text { toxicological effects } \\
\text { (Not teratogenic) }\end{array}$ \\
\hline \multirow{2}{*}{ Mouse } & $\begin{array}{l}90 \text {-day subacute } \\
\text { toxicity study }\end{array}$ & $\begin{array}{l}0,200,1,200,8,000 \mathrm{ppm} \\
\text { M: } 0,27,159,1,020 \\
\text { F: } 0,34,179,1,350\end{array}$ & $\begin{array}{l}\text { M: } 1,020 \\
\text { F: } 1,350\end{array}$ & $\begin{array}{l}\text { M: - } \\
\text { F: - }\end{array}$ & $\begin{array}{l}\text { F/M: No toxicological } \\
\text { effects }\end{array}$ \\
\hline & $\begin{array}{l}\text { 18-month } \\
\text { carcinogenicity } \\
\text { study }\end{array}$ & $\begin{array}{l}\text { 0, 200, 1,250, 8,000 ppm } \\
\text { M: } 0,22.7,140,884 \\
\text { F: } 0,31.6,186,1,320\end{array}$ & $\begin{array}{l}\text { M: } 884 \\
\text { F: } 1,320\end{array}$ & $\begin{array}{l}\text { M: - } \\
\text { F: - }\end{array}$ & $\begin{array}{l}\text { F/M: No toxicological } \\
\text { effects } \\
\text { (Not carcinogenic) }\end{array}$ \\
\hline Rabbit & $\begin{array}{l}\text { Developmental } \\
\text { toxicity study }\end{array}$ & $0,100,300,1,000$ & $\begin{array}{l}\text { Maternal: } 1,000 \\
\text { Embryo/fetus: } 1,000\end{array}$ & $\begin{array}{l}\text { Maternal: - } \\
\text { Embryo/fetus: - }\end{array}$ & $\begin{array}{l}\text { Maternal: No toxicologi- } \\
\text { cal effects } \\
\text { Embryo/fetus: No } \\
\text { toxicological effects } \\
\text { (Not teratogenic) }\end{array}$ \\
\hline \multirow[b]{2}{*}{ Dog } & $\begin{array}{l}90 \text {-day subacute } \\
\text { toxicity study }\end{array}$ & $\begin{array}{l}0,100,1,000,10,000 \mathrm{ppm} \\
\mathrm{M}: 0,2.68,26.8,266 \\
\text { F: } 0,2.75,26.9,270\end{array}$ & $\begin{array}{l}\text { M: } 2.68 \\
\text { F: } 26.9\end{array}$ & $\begin{array}{l}\text { M: } 26.8 \\
\text { F: } 270\end{array}$ & F/M: Increase in ALP \\
\hline & $\begin{array}{l}\text { One-year chronic } \\
\text { toxicity study }\end{array}$ & $\begin{array}{l}0,50,150,1,000 \\
10,000 \mathrm{ppm} \\
\mathrm{M}: 0,1.29,4.07,27.2,259 \\
\text { F: } 0,1.47,4.20,27.6,288\end{array}$ & $\begin{array}{l}\text { M: } 1.29 \\
\text { F: } 1.47\end{array}$ & $\begin{array}{l}\text { M: } 4.07 \\
F: 4.20\end{array}$ & F/M: Increase in ALP \\
\hline \multicolumn{3}{|c|}{ ADI } & \multicolumn{3}{|l|}{$\begin{array}{l}\text { NOAEL: } 1.29 \\
\text { SF: } 100 \\
\text { ADI: } 0.012\end{array}$} \\
\hline \multicolumn{3}{|c|}{ The critical study for setting ADI } & \multicolumn{3}{|c|}{ One-year chronic toxicity study in dogs } \\
\hline
\end{tabular}

M, Male; F, Female; F/M, both sexes; PM, Male in P (Parent) generation; PF, Female in P generation; $F_{1} M$, Male in $F_{1}$ generation; $F_{1} F$, Female in $\mathrm{F}_{1}$ generation; -, LOAEL was not derived; ${ }^{1)}$, The adverse effect observed at LOAEL; ADI, Acceptable daily intake; SF, Safety factor 